\title{
MicroRNA-10b Plays a Role in Bone Formation by Suppressing Interleukin-22 in Ankylosing Spondylitis
}

\author{
Pu-Reum Park, M.S. ${ }^{1}$, Sungsin Jo, Ph.D. ${ }^{2}$, So-Hee Jin, M.S. ${ }^{1}$, Tae-Jong Kim, Ph.D. ${ }^{1}$ \\ ${ }^{1}$ Department of Rheumatology, Research Institute of Medical Sciences, Chonnam National University Medical School and Hospital, Gwangju, \\ ${ }^{2}$ Department of Rheumatology, Hanyang University Hospital for Rheumatic Diseases, Seoul, Korea
}

\begin{abstract}
Objective. The microRNA (miR)-10b is the Thelper (Th) 17 cell specific in patients with ankylosing spondylitis (AS). The interleukin (IL)-22, which is closely related to Th17 cells, has been implicated in the regulation of new bone formation in experimental models. Therefore, the aim of this study was to evaluate whether miR-10b affects bone formation via the IL-22 pathway in AS. Methods. Primary CD4 + T cells from AS were purified and transfected with miR-10b, anti-miR-10b, or scramble. Cell-surface markers and cytokine expression were analyzed by flow cytometry and enzyme-linked immunosorbent assay. Primary bone-derived cells (BdCs) from the facet joints of the spine were isolated, then osteogenic differentiation of primary BdCs was performed. We assessed alkaline phosphatase (ALP) activity and staining of BdCs at early time points. Alizarin red $\mathrm{S}$ staining of BdCs was performed at late time points. Results. Overexpression of miR-10b reduced both IL-22 producing cell frequencies and cytokine production in T cells from the patients with AS. The IL-22 significantly increased ALP staining and bone mineralization. The ALP promotor activity of AS-BdCs was notably higher for the IL-22 concentration. The supernatants of the miR-10b overexpression group suppressed ALP activity on osteogenic progenitor cells from the facet joints of the spine in patients with AS. Conclusion. Our data suggest that miR-10b suppresses IL-22 production, which was involved in osteogenic proliferation in AS. Therefore, miR-10b might be a potential therapeutic candidate for regulation of new bone formation in patients with AS. (J Rheum Dis 2020;27:61-67)
\end{abstract}

Key Words. Ankylosing spondylitis, MicroRNA, Th17 cell, Osteogenesis

\section{INTRODUCTION}

Ankylosing spondylitis (AS) is characterized by inflammation of the axial skeleton, sacroiliac joints and, to a lesser degree peripheral arthritis and certain extra-articular organs, including the gastrointestinal tract, eyes, skin, and cardiovascular system [1].

AS is genetically associated with single nucleotide polymorphisms (SNPs) in the common p40 subunit of interleukin (IL)-12/23 and IL-23 receptor (IL-23R) SNPs. Recently, accumulating data suggest that the IL-23 pathway plays a main role in AS. Two of the major effector cytokines downstream of IL-23R are IL-17 and IL-22 [2]. The IL-17 producing Thelper (Th) 17 cells are significantly more prevalent in peripheral blood mononuclear cells (PBMCs) in patients with AS. Subsequent studies have revealed that the activation of Th17 cells is critical for inflammatory responses in AS [3-7]. The most characteristic feature in AS is subchondral eburnation and syndesmophytes that could lead to ankylosis and spinal fusion. Although the basis for the new bone formation in AS remains poorly understood, recently it was reported that IL-22 has been implicated in the regulation of new bone formation [8]. Moreover, it was suggested that human mesenchymal stem cell osteogenesis was enhanced following IL-22 exposure [9].

The microRNA (miR) is noncoding RNA oligonucleotide that has emerged as potent regulator of target gene

\footnotetext{
Received : October 6, 2019, Revised : December 2, 2019, Accepted : December 3, 2019

Corresponding to : Tae-Jong Kim (10 http://orcid.org/0000-0002-2871-1635

Department of Rheumatology, Research Institute of Medical Sciences, Chonnam National University Medical School and Hospital, 80 Deoknam-gil, Nam-gu, Gwangju 61748, Korea. E-mail : ktj1562@chonnam.ac.kr
}

Copyright (c) 2020 by The Korean College of Rheumatology. All rights reserved.

This is an Open Access article, which permits unrestricted non-commerical use, distribution, and reproduction in any medium, provided the original work is properly cited. 
expression [10]. They have been identified as appearing to be critical for fine-tuning many biological processes and offer the prospect of multiple targets [11,12]. Emerging data suggest that single miR species can alter the immune responses [13-15]. We recently reported a novel Th17 regulator miR-10b that is present in Th17 cells from patients with AS [16].

Th17 cells are the main producers of IL-22 [17], which was involved in osteogenesis in AS. Because we found that the main function of miR-10b is suppression of pro-inflammatory Th17 cell cytokines, we hypothesized that miR-10b may suppress production of IL-22 cytokines from Th17 cell and retard bone formation in AS.

\section{MATERIALS AND METHODS}

\section{Purification of primary CD4 + cell and transfection}

We obtained blood samples from six patients with AS (defined by modified NewYork criteria). This study was approved by the Institutional Review Board (IRB) of Chonnam National University Hospital (IRB no. CNUH2011-199) and Hanyang University Hospital (IRB no. 2014-05-002-001). Primary CD4+ T cells were purified using a negative selection by MACS CD4 $+\mathrm{T}$ cell isolation kit (Miltenyi Biotec, Bergisch Gladbach, Germany). We did transfection with a Neon transfection system device (Thermos Fisher Scientific, Waltham, MA, USA) according to the manufacturer's instructions. Briefly, CD4+ cells were stimulated for 19 hours with $2 \mathrm{ug} / \mathrm{mL}$ phytohaemagglutinin before transfection and resuspended in $\mathrm{T}$ buffer solution. $20 \mathrm{uM}$ oligonucleotides (including miR-10b mimic, anti-miR-10b, and miR-scramble) were added into the solution and mixed. Then the mixtures were transferred to electroporation cuvettes and placed in the transfection device. After transfection, cells were cultured in RPMI with $10 \%$ fetal calf serum. Transfected cells were then incubated under normal condition (humidified $37^{\circ} \mathrm{C} / 5 \% \mathrm{CO}_{2}$ ) until analysis.

\section{Human primary BdCs isolation and in vitro osteogenic differentiation}

Primary bone-derived cells (BdCs) were isolated and cultured as previously described [18]. Osteogenic differentiation of primary BdCs was performed using the previous method [19]. Briefly, the cells were seeded in growth medium and then stimulated with osteogenic conditional medium, including ascorbic acid, beta-glycerol phosphate, and dexamethasone. The osteogenic me- dium (OM) was changed every three days. We assessed alkaline phosphatase (ALP) activity and staining at early time points after stimulation with OM. Alizarin red S (ARS) staining was performed at late time points after the stimulation with OM.

\section{ALP promoter assay}

A plasmid with the ALP promoter was a gift from Prof. KwangYoul Lee (College of Pharmacy, Chonnam National University, Gwangju, Korea) [20]. The 293T cells were co-transfected with the ALP promoter plasmid and the firefly luciferase gene using Lipofectamine 3000, after which luciferase activity was assessed according to the manufacturer's protocol (E1500; Promega, Madison, WI, USA). Luciferase activity was measured with a luminometer (Berthold, Oak Ridge, TN, USA).

\section{Flow cytometry analysis}

Cell-surface markers and cytokine expression were detected by flow cytometry after re-stimulation with PMA and ionomycin in the presence of brefeldin A. The antibodies used for the surface phenotype, allophycocyanin-labeled anti-CD4 (Becton Dickinson, San Diego, CA, USA), and phycoerythrin-Cy7-labeled anti-IL-22 (Becton Dickinson, San Diego, CA, USA). Flow cytometric data were collected on an LSRII (BD PharMingen, San Jose, CA, USA); a minimum of $1 \times 10^{5}$ events were collected. Data were analyzed using FlowJo software (Tree Star, Ashland, OR, USA).

\section{Detection of cytokines in cell culture supernatant} The sample was collected and transferred into a single well of a cytokine assay plate supplied by the manufacturer (Ebioscience, San Diego, CA, USA), followed by washing and binding with the primary and secondary antibodies. The secondary antibody contained a horseradish peroxidase enzyme that can metabolize the substrate to turn it blue color. The color changes to yellowbrown upon addition of the stop reaction buffer. The color intensity representing the level of cytokine expression was measured in the plate reader at $405 \mathrm{~nm}$. A standardized column for each cytokine was set in each assay plate to convert the light absorbance from each sample into the weight per $\mathrm{mL}$ of medium.

\section{Statistical analysis}

Statistical analysis was performed using GraphPad Prism software version 5 (GraphPad, Inc., San Diego, CA, 
USA). A p-value less than 0.05 was considered statistically significant.

\section{RESULTS}

miR-10b suppresses IL-22 cytokine production

We first investigated the effects of miR-10b on IL-22 production in AS. CD4+T cells from PBMCs of AS were isolated and transfected with miR-10b mimic, anti-miR-10b, or scramble as a control. The cell viability did not affected by miR-10b transfection (Supplementary Figure 1). After 72 hours culture, a representative figure of flow cytometry results was shown for each group (Figure 1A). Overexpression of miR-10b reduced IL-22
A

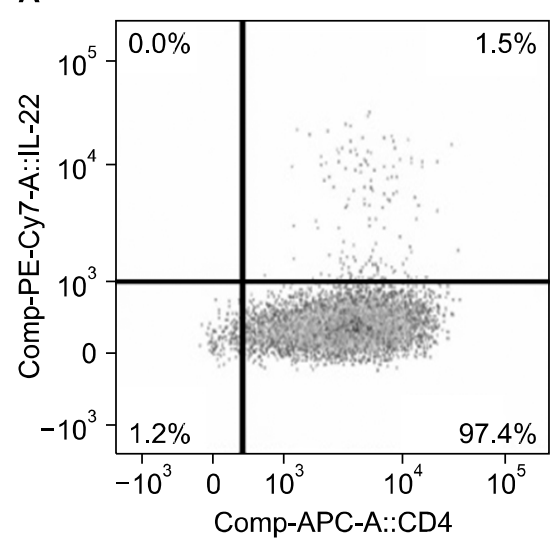

B
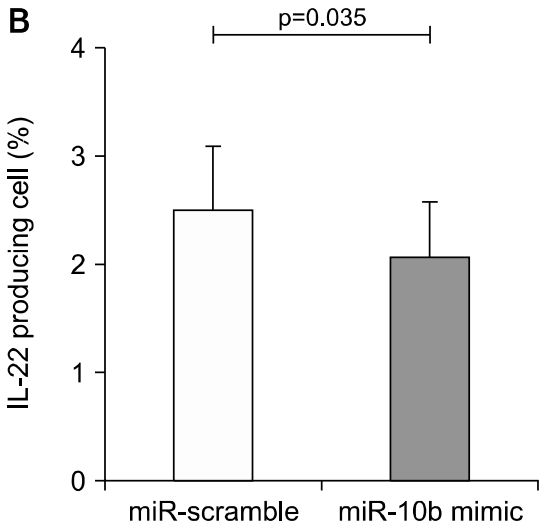

C

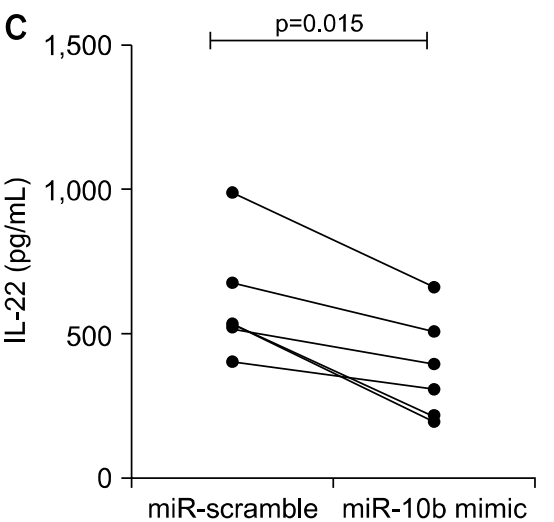

miR-10b mimic
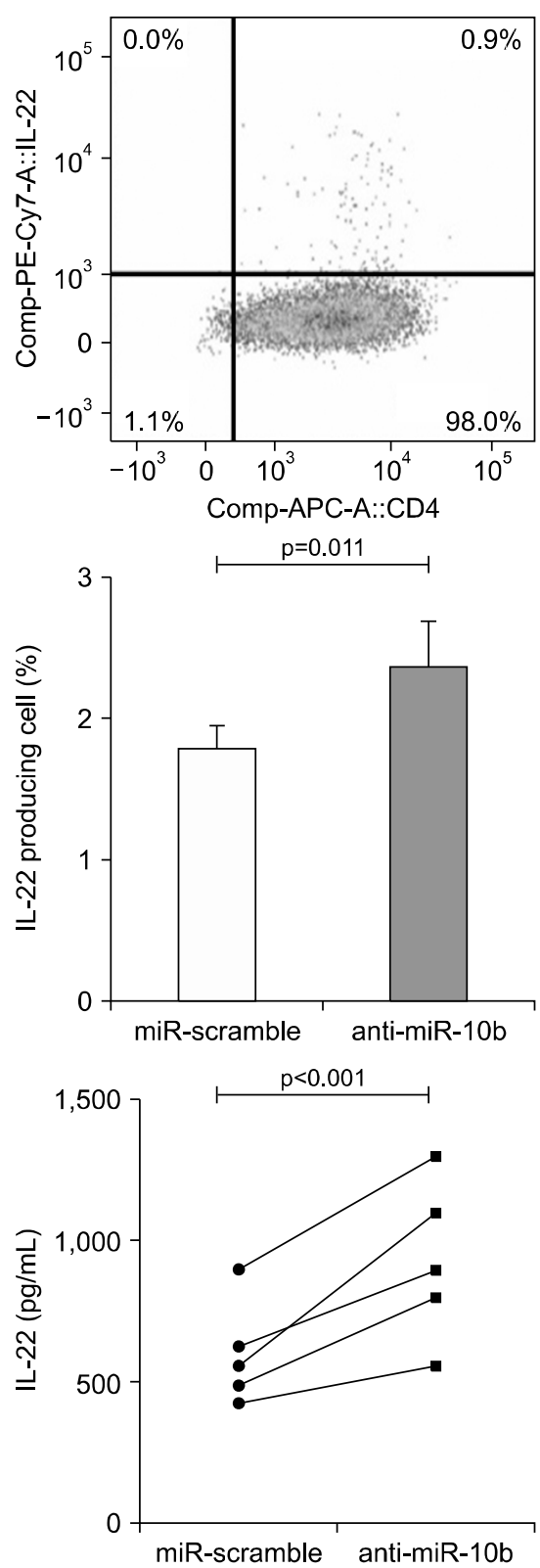

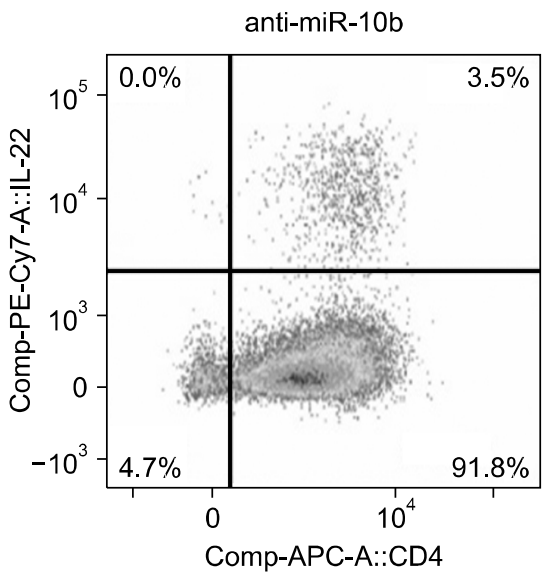

Figure 1. MicroRNA (miR)-10b suppresses interleukin (IL)-22 cytokine production. Frequency of IL-22 producing cells and production of IL-22 in CD4 T cells from ankylosing spondylitis (AS) transfected with miR-10b mimic, anti-miR-10b, or miR-scramble and stimulated with anti-CD2/3/28-coated beads for 72 hours. A representative flow cytometry result is shown for each group (A). Overexpression of miR-10b reduced IL-22 producing cell frequencies and cytokine production in T cells from the patients with AS. Whereas, inhibition of miR-10b significantly increased the expression of IL-22 cytokine (B, C). APC: allophycocyanin, PE-Cy7: phycoerythrin-Cy7. 
producing cell frequencies $(2.49 \pm 1.56$ vs. $2.04 \pm 1.38$, $\mathrm{p}=0.035$ ) (Figure 1B, left panel). Silencing of miR-10b resulted in reduced levels of IL-22 production, verified by decreased in the proportion of IL-22 positive cell (1.78 \pm 0.37 vs. $2.16 \pm 0.38, p=0.011$ ) (Figure $1 B$, right panel). miR-10b overexpression markedly decreased IL-22 cytokine production in $\mathrm{T}$ cells from the patients with AS $(600.3 \pm 189.9$ vs. $387.7 \pm 163.9, p=0.015)$, whereas inhibition of miR-10b significantly increased the expression of IL-22 cytokine (601.5 \pm 182.6 vs. $932.0 \pm 283.1, \mathrm{p}<$ 0.001) (Figure 1C, right panel).

\section{IL-22 enhances osteogenic activity and differentiation in AS}

It has been reported that the levels of secreted and anchored ALP were elevated in AS-BdCs [21]. We wondered whether IL-22 can induce osteogenic activity in AS. Therefore, we stimulated AS-BdCs with IL-22 in a dose-dependent manner $(0,5,10,20 \mathrm{ng} / \mathrm{mL})$. We finally observed that IL-22 significantly increased ALP staining and mineralization as assessed by ARS staining (Figure $2 \mathrm{~A}$ ). The ALP promotor activity of AS-BdCs was notably higher for the IL-22 concentration up to $20 \mathrm{ng} / \mathrm{mL}$ (Figure 2B). Furthermore, adding IL-22 exhibited induced intercellular ALP activity (Figure 2C).

\section{miR-10b suppresses ALP activity on osteoprogenitor cells in AS}

We collected supernatants from three-days cultured $\mathrm{T}$ cells, which had been transfected with miR-10b mimic or scramble. Then we added these supernatants to the AS-BdCs to see whether the miR-10b affected osteogenic

A

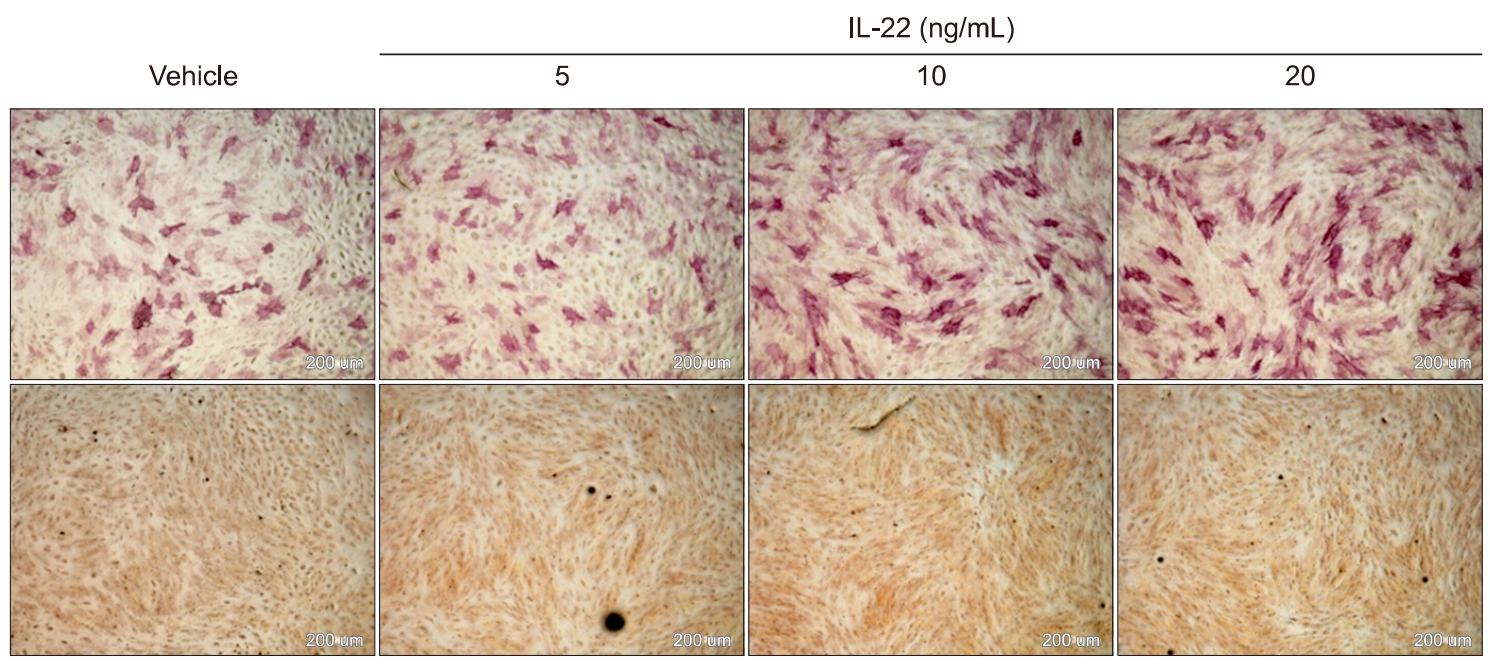

B

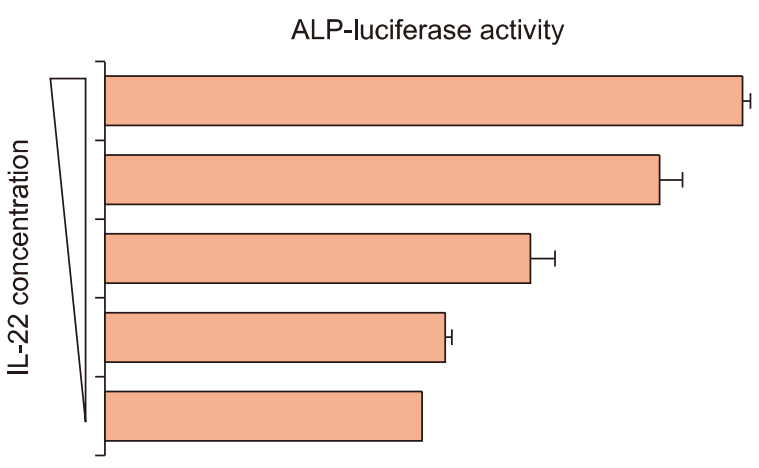

C

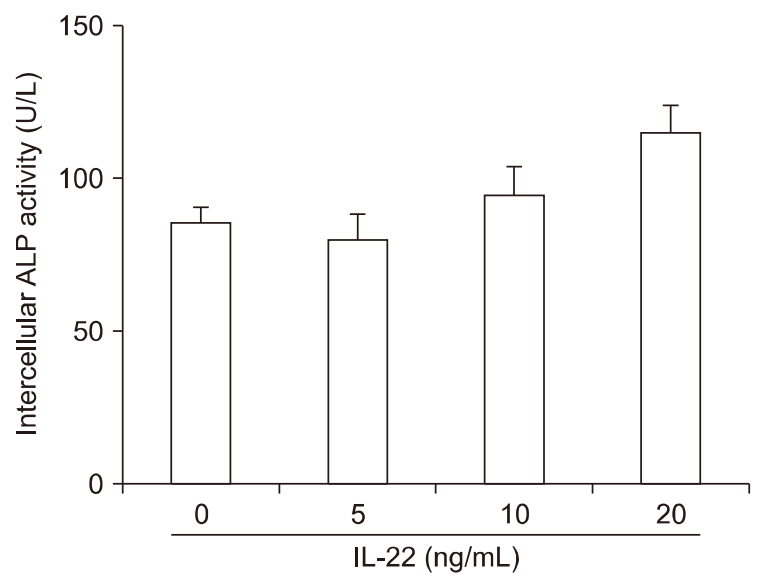

Figure 2. Interleukin (IL)-22 promotes osteoblastogenesis. Primary bone-derived cells (BdCs) from ankylosing spondylitis (AS) were isolated and cultured. Alkaline phosphatase (ALP) activity and staining were assessed at early time points after stimulation with osteogenic medium (OM). Alizarin red S (ARS) staining was done at late time points after stimulation with OM (A). The ALP promotor and intercellular ALP activity of AS-BdCs depending on IL-22 concentration were measured (B, C). 


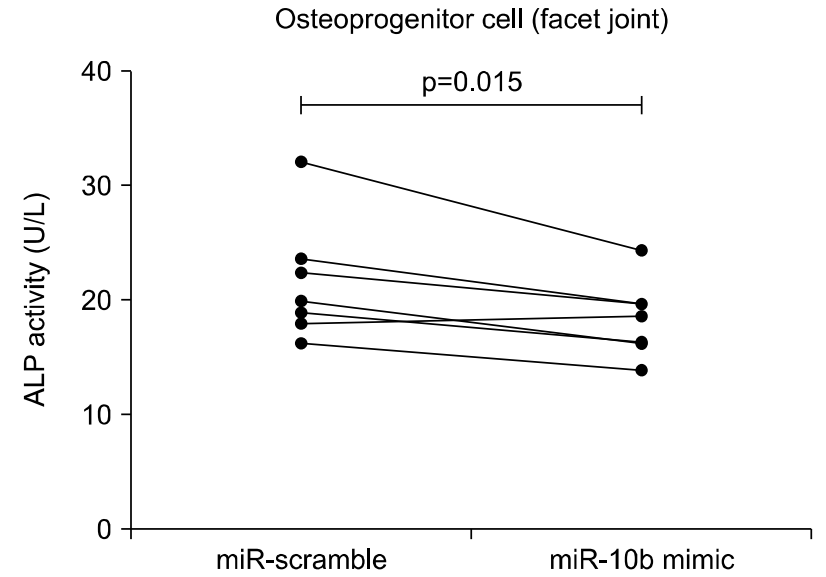

Figure 3. MicroRNA (miR)-10 suppresses alkaline phosphatase (ALP) activity on osteoprogenitor cells in ankylosing spondylitis (AS). Supernatants from T cells, which had been transfected with miR-10b mimic or scramble, were added on the primary bone-derived cells bone-derived cells from the facet joint in AS.

activity. We found that supernatants of the miR-10b overexpression group suppressed ALP activity on osteogenic progenitor cells from the facet joints of the spine in patients with AS $(21.67 \pm 4.74$ vs. $18.50 \pm 3.37 \mathrm{p}=0.015)$ (Figure 3).

\section{DISCUSSION}

The AS shows a propensity for new bone formation following inflammation. Bone formation occurs at sites of the entheseal region and histologically may exhibit endochondral, intramembranous or chondroidal metaplasia [22]. Although the mechanism for the new bone formation in AS remains poorly understood, elevated ALP levels in serum were observed in patients with AS. Moreover, ALP secretion and levels of anchored ALP were high in AS-BdCs compared with healthy control-BdCs [21]. In this study, we isolated and cultured primary BdCs from surgically obtained bone from patients with AS. Because AS-BdCs exhibited dramatically increased ALP activity and mineralization, which are both features of terminally differentiated osteoblasts, AS-BdCs can be suitable samples for experiment of bone formation in AS.

Human MSC may play a major role in both bone repair and aberrant new bone formation at the enthuses [23]. IL-22 regulates stem cell function in the liver, skin, and intestine has been dubbed a master regulator of stem cell function [24]. One study has found that IL-22 was involved in human MSC proliferation and migration in in- flammatory environments [9]. Sherlock and his colleague tested whether IL-22 could have an osteoproliferative effect using an experimental model for sponsyloarthritis. Osteoblasts from MSC are central to the formation of new bone, IL-22 more effectively induces genes that regulate bone formation, specifically those that encode Wnt family members, bone morphogenic proteins and alkaline phosphatase. Taken together, these results suggest that the osteoprolifera $\neg$ tive component of disease is mediated by IL-22, promoting entheseal and periosteal bone formation [8]. Based on these observations, we hypothesize that IL-22 may also regulate human MSC function, particularly MSC osteogenesis in an inflammationdependent context. According to the data of ALP promotor activity and mineralization of AS-BdCs, IL-22 can induce osteogenic activity in patients with AS. In line with previous results, we also concluded that IL-22 induced osteogenesis in AS-BdCs.

Although there has been interest in the progression of structural damage in AS, only limited and inconsistent data are available about the treatment for structural damage $[25,26]$. We previously identified AS Th17-specific miRs and described the function of miR-10b, in the negative regulation of inflammatory cytokine productions by Th17 cells, using RNA sequencing. miR-10b was upregulated in AS Th17 cells, where it likely functions to suppress IL-17A production. Previous researches have linked miR-10b to cancer pathogenesis. It is up-regulated in metastatic breast cancer, where it down-regulates Homeobox D10 [27], and can inhibit cell proliferation and enhance apoptosis in gastric cancer cell lines [28]. However, we reported that miR-10b can inhibit MAP3K7 expression in CD4 $\mathrm{T}$ cells and that this can lead to inhibition of Th17 cell cytokine (without significant effects on cell viability) [16]. MAP3K7 activates NF- $\kappa$ B signal transduction by causing I $\kappa$ B degradation [29]. NF- $\kappa \mathrm{B}$ has been shown to be involved in the regulation inflammatory gene expression in Th17 cells, suggesting inhibition of NF $\kappa \mathrm{B}$ as a downstream mechanism for miR$10 \mathrm{~b}$ regulation of Th17 function. It is possible that suppression of MAP3K7 as a target of miR-10b resulted in decrease of IL-22 cytokines. In this study, miR-10b overexpression reduced both IL-22 producing cell frequencies and IL-22 production in T cells from the patients with AS. Therefore we did further experiments using Th17 cell specific miR-10b, focusing on treatment for bony progression in AS. In line with the miR-10b effect on IL-22, miR-10b overexpression suppresses osteoblastic activity 
in patients with AS by suppressing IL-22 production. For the suppression of osteogenic IL-22, gene therapy using miR-10b might be a potential candidate for controlling inflammation and delaying bony progression in patients with AS.

There are some limitations to this study. Because the osteogenic effect of miR-10b was shown only by ALP activity, experiments about mineralization or calcification by miR-10b on AS-BdCs should be carried out. IL-22 blocking experiment should be carried out to verify the IL-22 effects on osteogenesis by miR-10b. All experiments were carried out in vitro. Further in vivo studies should be done considering not only IL-22 but also other confounding factors.

However our data suggest that miR-10b has preventive effect on osteoblastic activity in AS via suppressing IL-22 cytokines. Therefore, the gene based novel therapy can be a candidate for treatment of inflammation and bony progression in AS.

\section{CONCLUSION}

In the present study, miR-10b overexpression reduced both IL-22 producing cell frequencies and IL-22 production in T cells from the patients with AS. miR-10b overexpression suppresses osteoblastic activity in patients with AS by suppressing IL-22 production. Therefore, miR-10b might be a potential therapeutic candidate for controlling inflammation and regulation of new bone formation in patients with AS.

\section{CONFLICT OF INTEREST}

No potential conflict of interest relevant to this article was reported.

\section{AUTHOR CONTRIBUTIONS}

T.J.K. designed the study. T.J.K., P.R.P., and S.J. drafted the manuscript. P.R.P. and S.H.J. contributed to the acquisition and analysis of data. All authors approved the final manuscript.

\section{SUPPLEMENTARY DATA}

Supplementary data can be found with this article online at https://doi.org/10.4078/jrd.2020.27.1.61.

\section{REFERENCES}

1. Khan MA, van der Linden SM. A wider spectrum of spondyloarthropathies. Semin Arthritis Rheum 1990;20: 107-13.

2. Zheng Y, Danilenko DM, Valdez P, Kasman I, EasthamAnderson J, Wu J, et al. Interleukin-22, a T(H) 17 cytokine, mediates IL-23-induced dermal inflammation and acanthosis. Nature 2007;445:648-51.

3. Bowness P, Ridley A, Shaw J, Chan AT, Wong-Baeza I, Fleming M, et al. Th17 cells expressing KIR3DL2+ and responsive to HLA-B27 homodimers are increased in ankylosing spondylitis. J Immunol 2011;186:2672-80.

4. Jandus C, Bioley G, Rivals JP, Dudler J, Speiser D, Romero P. Increased numbers of circulating polyfunctional Th17 memory cells in patients with seronegative spondylarthritides. Arthritis Rheum 2008;58:2307-17.

5. Xueyi L, Lina C, Zhenbiao W, Qing H, Qiang L, Zhu P. Levels of circulating Th17 cells and regulatory T cells in ankylosing spondylitis patients with an inadequate response to anti-TNF- $\alpha$ therapy. J Clin Immunol 2013;33:151-61.

6. Bidad K, Salehi E, Jamshidi A, Saboor-Yaraghi AA, Oraei M, Meysamie A, et al. Effect of all-transretinoic acid on Th17 and $\mathrm{T}$ regulatory cell subsets in patients with ankylosing spondylitis. J Rheumatol 2013;40:476-83.

7. Shen H, Goodall JC, Hill Gaston JS. Frequency and phenotype of peripheral blood Th17 cells in ankylosing spondylitis and rheumatoid arthritis. Arthritis Rheum 2009;60:164756.

8. Sherlock JP, Joyce-Shaikh B, Turner SP, Chao CC, Sathe M, Grein J, et al. IL-23 induces spondyloarthropathy by acting on ROR- $\gamma \mathrm{t}+\mathrm{CD} 3+\mathrm{CD} 4-\mathrm{CD} 8$ - entheseal resident T cells. Nat Med 2012;18:1069-76.

9. El-Zayadi AA, Jones EA, Churchman SM, Baboolal TG, Cuthbert RJ, El-Jawhari JJ, et al. Interleukin-22 drives the proliferation, migration and osteogenic differentiation of mesenchymal stem cells: a novel cytokine that could contribute to new bone formation in spondyloarthropathies. Rheumatology (Oxford) 2017;56:488-93.

10. Bartel DP. MicroRNAs: genomics, biogenesis, mechanism, and function. Cell 2004;116:281-97.

11. O'Connell RM, Rao DS, Chaudhuri AA, Baltimore D. Physiological and pathological roles for microRNAs in the immune system. Nat Rev Immunol 2010;10:111-22.

12. Pillai RS, Bhattacharyya SN, Filipowicz W. Repression of protein synthesis by miRNAs: how many mechanisms? Trends Cell Biol 2007;17:118-26.

13. Xiao C, Srinivasan L, Calado DP, Patterson HC, Zhang B, Wang $\mathrm{J}$, et al. Lymphoproliferative disease and autoimmunity in mice with increased miR-17-92 expression in lymphocytes. Nat Immunol 2008;9:405-14.

14. Jin HM, Kim TJ, Choi JH, Kim MJ, Cho YN, Nam KI, et al. MicroRNA-155 as a proinflammatory regulator via SHIP-1 down-regulation in acute gouty arthritis. Arthritis Res Ther 2014; 16:R88.

15. Thai TH, Calado DP, Casola S, Ansel KM, Xiao C, Xue Y, et al. Regulation of the germinal center response by microRNA155. Science 2007;316:604-8.

16. Chen L, Al-Mossawi MH, Ridley A, Sekine T, Hammitzsch A, de Wit J, et al. miR-10b-5p is a novel Th17 regulator pres- 
ent in Th17 cells from ankylosing spondylitis. Ann Rheum Dis 2017;76:620-5.

17. Liang SC, Tan XY, Luxenberg DP, Karim R, DunussiJoannopoulos K, Collins M, et al. Interleukin (IL)-22 and IL-17 are coexpressed by Th17 cells and cooperatively enhance expression of antimicrobial peptides. J Exp Med 2006;203:2271-9.

18. Jo S, Koo BS, Lee B, Kwon E, Lee YL, Chung H, et al. A novel role for bone-derived cells in ankylosing spondylitis: focus on IL-23. Biochem Biophys Res Commun 2017;491:787-93.

19. Jo S, Kang S, Han J, Choi SH, Park YS, Sung IH, et al. Accelerated osteogenic differentiation of human bone-derived cells in ankylosing spondylitis. J Bone Miner Metab 2018;36:307-13.

20. Choi YH, Kim YJ, Jeong HM, Jin YH, Yeo CY, Lee KY. Akt enhances Runx2 protein stability by regulating Smurf2 function during osteoblast differentiation. FEBS J 2014;281: 3656-66.

21. Jo S, Wang SE, Lee YL, Kang S, Lee B, Han J, et al. IL-17A induces osteoblast differentiation by activating JAK2/STAT3 in ankylosing spondylitis. Arthritis Res Ther 2018;20:115.

22. Benjamin M, Toumi H, Suzuki D, Hayashi K, McGonagle D. Evidence for a distinctive pattern of bone formation in enthesophytes. Ann Rheum Dis 2009;68:1003-10.

23. Lories RJ, Derese I, Luyten FP. Modulation of bone morpho- genetic protein signaling inhibits the onset and progression of ankylosing enthesitis. J Clin Invest 2005;115:1571-9.

24. Feng D, Kong X, Weng H, Park O, Wang H, Dooley S, et al. Interleukin-22 promotes proliferation of liver stem/progenitor cells in mice and patients with chronic hepatitis B virus infection. Gastroenterology 2012;143:188-98.e7.

25. Baraliakos X, Haibel H, Listing J, Sieper J, Braun J. Continuous long-term anti-TNF therapy does not lead to an increase in the rate of new bone formation over 8 years in patients with ankylosing spondylitis. Ann Rheum Dis 2014;73:710-5.

26. Poddubnyy D, Rudwaleit M, Haibel H, Listing J, MärkerHermann E, Zeidler $\mathrm{H}$, et al. Effect of non-steroidal anti-inflammatory drugs on radiographic spinal progression in patients with axial spondyloarthritis: results from the German Spondyloarthritis Inception Cohort. Ann Rheum Dis 2012;71:1616-22.

27. Ma L, Teruya-Feldstein J, Weinberg RA. Tumour invasion and metastasis initiated by microRNA-10b in breast cancer. Nature 2007;449:682-8.

28. Li Z, Lei H, Luo M, Wang Y, Dong L, Ma Y, et al. DNA methylation downregulated mir-10b acts as a tumor suppressor in gastric cancer. Gastric Cancer 2015;18:43-54.

29. Wang C, Deng L, Hong M, Akkaraju GR, Inoue J, Chen ZJ. TAK1 is a ubiquitin-dependent kinase of MKK and IKK. Nature 2001;412:346-51. 